\title{
DETECÇÃO DE INCIDENTES EM UM TÚNEL URBANO CONGESTIONADO: UM ESTUDO DE CASO SOBRE O TÚNEL REBOUÇAS NA CIDADE DO RIO DE JANEIRO
}

\author{
M.L.B.Baltar ${ }^{\text {** }}$; P.C.M..Ribeiro ${ }^{1}$ \\ 1 Universidade Federal do Rio de Janeiro, 21941-914, Rio de Janeiro-RJ, Brasil \\ *mabaltar@gmail.com
}

Artigo submetido em 26/07/2017 e aceito em 05/10/2017

\section{RESUMO}

O objetivo desta pesquisa foi desenvolver um método teórico que elimine o grande número de alarmes falsos resultantes de paradas por congestionamento nos sistemas de detecção automática de incidentes instalados em túneis urbanos com grande fluxo. Esse método levou em consideração o conceito de ondas de choque com a finalidade de prever o momento que essa onda atingirá um determinado ponto no túnel. Após o desenvolvimento dessa metodologia, foi realizado um estudo de caso no Túnel Rebouças, localizado na Cidade do Rio de Janeiro. Nesse túnel há instalado um sistema de detecção automática de incidente que gera diversos alarmes falsos no horário de pico. Em cima das observações do Túnel Rebouças, tonou-se, como resultado, que é possível reduzir a taxa de alarmes falsos a partir da previsão das ondas de choque que atingem as galerias do túnel.

\section{INCIDENT DETECTION OF CONGESTIONED URBAN TUNNELS: A CASE STUDY OF REBOUÇAS TUNNEL IN RIO DE JANEIRO}

\begin{abstract}
The objective of this research is to develop a theoretical method capable of eliminating the large number of false alarms resulted of traffic stopping on the automatic incident detection system installed in urban tunnels with large traffic flow. This method took in consideration the concept of shocks wave and have with the purpose of predicting the moment that this wave will reach a certain point in the tunnel. After the development of the method,
\end{abstract}

a case study was conducted in the tunnel Rebouças, in Rio de Janeiro city, where there is an automatic incident detection system that generates a large number of false alarms during rush hours. With the suggested methodology, it was observed that it is possible to lower the number of false alarms by predicting the shock waves that hit the tunnel galleries.

KEYWORDS: Incidents, Urban Tunnel, Intelligent transport systems. 


\section{INTRODUÇÃO}

Os incidentes geram congestionamento e prejudicam o fluxo de tráfego resultando em uma falta de eficiência nos sistemas de transporte. O HCM (2010) define um incidente como sendo qualquer ocorrência em uma via que impeça o fluxo de tráfego normal. Coelho (2009) afirma que incidentes reduzem a capacidade das vias em absorver o fluxo passante e produz congestionamento em função da demanda e da oferta, ou seja, a consequência devido aos incidentes depende do volume de fluxo naquele determinado horário. Portanto, nos períodos de pico essas consequências são observadas mais rapidamente, pois a demanda por viagens é maior.

Lou et al.(2011) afirmam que, na prática, para aliviar os congestionamentos causados por incidentes, é necessária a detecção, resposta e remoção dos mesmos com o objetivo de reestabelecer a capacidade das vias da forma mais segura e rápida possível. No caso de vias expressas, em especial, os efeitos dos incidentes geralmente são mais graves, já que essas vias são caracterizadas por um grande fluxo de veículos em alta velocidade, resultando geralmente em incidentes com ferimentos graves ou, até mesmo, fatalidades (JEONG et al., 2011).

O gerenciamento de incidentes busca reestabelecer a capacidade da via no menor tempo possível. Sendo assim, quando ocorre um incidente, o tempo estimado para solucioná-lo é a chave do gerenciamento de incidente. Essa previsão pode ajudar os controladores a passar informações corretas para os usuários das vias, aplicando as soluções apropriadas de controle de tráfego na via do incidente e em suas proximidades, avaliando o efeito causado por essas intervenções.

Na prática, segundo Valenti et al. (2010), essa duração é estimada pelos controladores de tráfego baseados em sua experiência e no reconhecimento das características do incidente (sua natureza, se houve fatalidade, número de veículos envolvidos, etc.). A confiança nessas estimativas ainda é desconhecida e depende em larga escala da habilidade do operador. Resumidamente, Zheng et al. (2011) afirmam que os incidentes contribuem significativamente para os atrasos e custos no trânsito e sua detecção e limpeza feitas de forma rápida são meios mais efetivos de reduzir os impactos desses eventos não recorrentes.

Esta pesquisa tem como objetivo desenvolver um método teórico que reduza o grande número de alarmes falsos gerados pelos sistemas de detecção de incidentes quando a via está congestionada. $\mathrm{O}$ estudo foca especificamente em túneis urbanos congestionados.

Nas vias expressas, as filas não são estáticas como no caso dos cruzamentos. Nelas os veículos movem-se lentamente, com períodos de parada e de movimento (HCM, 2010). Nesses casos, o fluxo de tráfego sofre interrupções frequentes devido à falta de fluidez, consequentemente, os parâmetros de tráfego sofrem modificações bruscas. Portanto, os sistemas de detecção automática de incidentes (AID - Automatic Incident Detection) que utilizam apenas essas modificações dos parâmetros de tráfego para identificar um incidente geram um grande número de alarmes falsos. O sistema entende a interrupção do tráfego, que ocorre devido à falta de fluidez, como um incidente e não como uma situação recorrente de uma via opera em nível de serviço $\mathrm{F}$, que tem como característica o stop-and-go.

O método proposto trabalha com o conceito de ondas de choque para prever os momentos em que ocorrerão modificações bruscas nos parâmetros de tráfego sem ter ocorrido um incidente no trecho em questão. Essas ondas de choque iniciam na jusante do túnel e se alastram para a montante da via devido às perturbações de tráfego ocorridas nos horários de pico da via.

Pretende-se com esse método ter um sistema mais confiável, buscando, assim, soluções mais eficientes nos casos dos incidentes em túneis urbanos congestionados, reduzindo os atrasos, a deseconomia e o tamanho das filas causadas por eles. 
A FHWA (2010) mostra que as melhorias no gerenciamento de incidentes ajudam tanto a reduzir a duração dos incidentes em geral como os acidentes secundários. O impacto da redução de duração do incidente foi apresentado pelo Journal ITS apud FHWA (2010), que estimou que a probabilidade de ocorrer um acidente secundário aumenta $2,8 \%$ a cada minuto de duração do incidente principal.

Portanto, o tempo de detecção é o parâmetro mais importante no processo de gerenciamento de incidentes. Segundo Skorput et al (2010), para obter a máxima eficiência nesse gerenciamento, o tempo de detecção deve ser o mais curto possível, pois atrasos na detecção resultam em filas, congestionamentos e incidentes secundários.

\section{MATERIAIS E MÉTODOS}

\subsection{Ondas de choque}

Segundo o TRB (1976), uma onda de choque ocorre devido à descontinuidade do fluxo ou da densidade. A decorrência física dessa descontinuidade é a mudança abrupta da velocidade do carro, que pode causar tanto a aceleração do fluxo quanto sua desaceleração.

Isto é, uma onda de choque causa uma mudança descontínua nos parâmetros de tráfego dos veículos na via, já que as trajetórias que são atravessadas pela onda mudam de velocidade abruptamente (Immers \& Logghe, 2002).

Elas podem ser ocasionadas pela falta de capacidade da via, que pode ocorrer devido a incidentes, devido apenas a um fluxo maior que a capacidade ou um estreitamento, devido à redução do número de faixas, entre outros.

A velocidade da onda de choque $\left(u_{k}\right)$ é definida pela Equação (1).

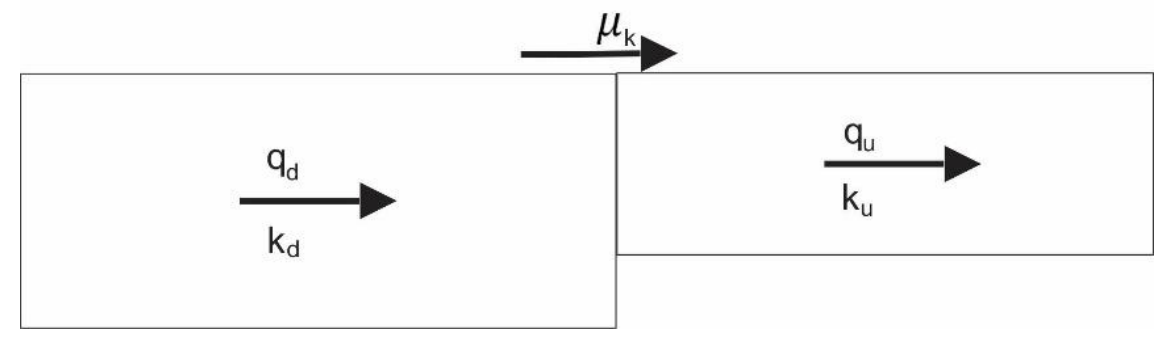

Figura 1- Variáveis para cálculo da velocidade da onda de choque Fonte: Garber et al (1996)

$$
u_{k}=\frac{q_{d}-q_{u}}{k_{d}-k_{u}}
$$

Onde, como mostrado na Figura 1,

$\mathrm{q}_{\mathrm{d}}$ fluxo da montante;

$\mathrm{q}_{\mathrm{u}}$ : fluxo da jusante;

$\mathrm{k}_{\mathrm{d}}$ : densidade da montante;

$\mathrm{k}_{\mathrm{u}}$ "densidade da jusante. 
Com base na Equação 1 é possível observar que a onda de choque pode se mover para frente ou para trás, já que não há uma regra. Quando a velocidade da onda de choque for positiva, ela se move na direção do fluxo, já quando for negativa essa onda se move no sentido contrário.

A Figura 2 mostra graficamente como a velocidade da onda é estimada, pelo declive da linha tracejada que liga o fluxo do gargalo as suas respectivas capacidades.

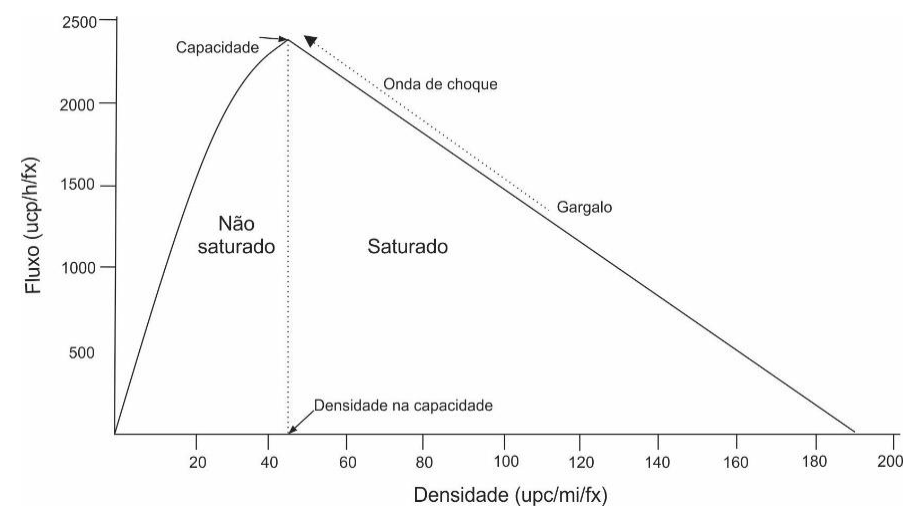

Figura 2- Relação fluxo e velocidade em uma onda de choque FONTE: HCM (2010)

Quando a velocidade das ondas de choque está no sentido do fluxo, as ondas passam a ser de recuperação. Quando ocorrem essas ondas, é possível notar a dissipação das filas acumuladas na sobre demanda. Nessas ondas, o sistema viário ainda opera com utilização intensa, já que não há recuperação imediata no tráfego.

A velocidade da onda de recuperação é obtida da mesma maneira que a da onda de choque. Entretanto, o fluxo e a densidade a serem considerados para comparação são os do trecho em que ainda há corrente de tráfego.

A fila frontal de um segmento tem, portanto, a densidade reduzida durante a recuperação, enquanto o fim da fila ainda sofre efeito da onda de choque.

\subsection{Sistemas de detecção automática de incidentes (AID)}

Os AID (Automatic Incident Detection) são apoios para o gerenciamento e ajudam a reduzir os congestionamentos de tráfego e os acidentes secundários quando aplicados de forma correta.

Os métodos de detecção de incidentes incluem telefone particular, call box, frota operacional, chamadas de emergência, imagens/vídeo e sistemas de detecção automática. Para reduzir o número de alarmes falsos, muitas vezes são utilizados sistemas de verificação que checam/determinam a exata posição e a natureza do incidente. Essa verificação pode ser feita pelas câmeras ou pelo cruzamento das informações fornecidas pela população (Skorput et al., 2010).

A Figura 3 apresenta as modificações na velocidade e na densidade quando ocorre um incidente em uma via. Antes do ponto em que ocorreu o incidente, a velocidade diminui e a densidade aumenta. Logo após o local do incidente, o inverso acontece, a velocidade aumenta e a densidade diminui. 


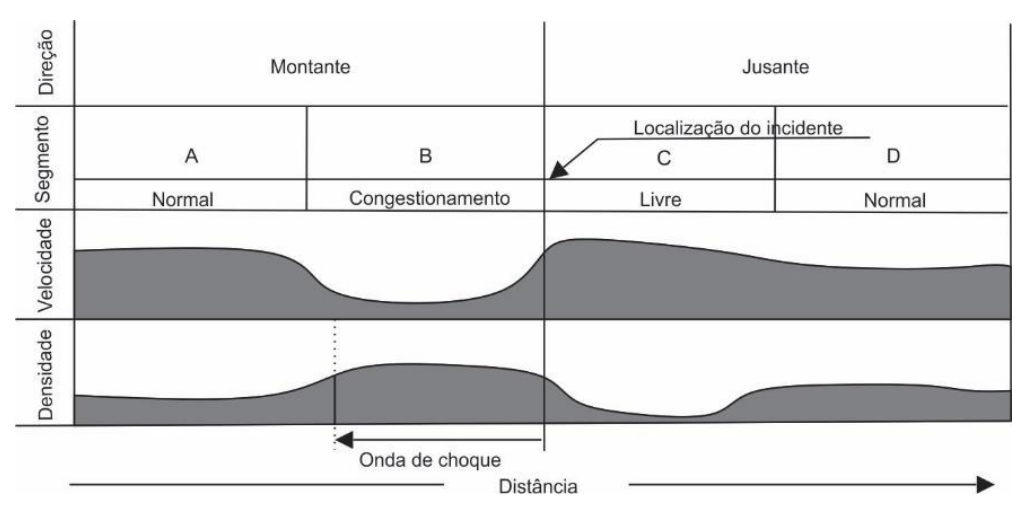

Figura 3- Características do tráfego durante um incidente FONTE: Skorput et al.(2010)

Todos os métodos pesquisados trabalham com pelo menos um dos parâmetros de tráfego: velocidade, densidade e fluxo, e detectam o incidente através do processamento das imagens ou de comparação entre séries temporais. Assim, eles buscam menor tempo de detecção e menor taxa de alarmes falsos.

A grande diferença entre os métodos é a forma de obtenção de dados, podendo utilizar: câmeras de vídeo, detectores de laços indutivos e veículos de teste. Esse último vem sendo utilizado de forma integrada com outros detectores a fim de reduzir o número de alarmes falsos.

Chen et al. (2010) buscam um algoritmo mais simples e utilizam os dados como velocidade, ocupação e fluxo que são coletados por meio de laços indutivos e analisa os parâmetros de tráfego fazendo uma comparação dos dados coletados a montante e a jusante da via. Quando há uma mudança brusca dos dados coletados, como uma diminuição do fluxo e da velocidade e um aumento da densidade, ele mostra que alguma situação anormal está ocorrendo. Comparado com redes neurais artificiais, ele é mais eficiente no caso de alarmes falsos (1,03\% em comparação a $1,22 \%$ no ANN) e demora menos tempo na detecção (41 segundos em comparação com 91segundos no ANN).

Já Wang (2011) propôs um método que utiliza um detector de veículos na coleta de informações relevantes e processa esses dados para que sejam classificados por uma rede neural. Seis informações entram no sistema através dessa rede, são elas: o fluxo médio, espaço e velocidade dos pontos A e B. Depois de repetidos testes que combinam esses parâmetros na camada oculta da rede neural, apenas um nó é gerado com os avisos de estado normal ou anormal do tráfego. Esse algoritmo teve uma maior taxa de detecção, menor número de alarmes falsos e um tempo médio de detecção intermediário, após o autor comparar o algoritmo desenvolvido com algoritmos tradicionais: o California \#7 algorithm e o Mcmaster algorithm.

Lu et al. (2012) desenvolveram um estudo preliminar que indica a programação lógica indutiva (ILP), como um método de AID competitivo e promissor. Esse método tem a vantagem de relacionar dados mais complexos. Esse método usa uma coleção de dados obtidos de sensores das vias expressas e compara-os com os dados em tempo real, como velocidade, volume e ocupação, definindo se há ou não um incidente naquela via.

Jeonget al. (2011) propõem um novo algoritmo utilizando um sistema baseado em frequência de loops, que necessita tanto dos dados de ocupação como velocidade da via. Com um intervalo de 30 segundos, o programa obtém a ocupação e a velocidade dos dois sentidos da via, analisa esses dados e observa se houve oscilações, comparando-os com os dos dados anteriormente colhidos. Um diferencial é que os dados de comparação são adaptados de acordo com o fluxo do tráfego, obtendo assim um número menor de alarmes falsos. 
Jiang et al. (2010) propuseram um algoritmo que detecta um incidente a partir das mudanças dos parâmetros de tráfego, os quais passam a não seguir mais a tendência que vinha sendo imposta. Os dados foram agrupados em três escalas de tempo: 20 segundos, 1 minuto e 5 minutos. $\mathrm{O}$ problema é que algumas mudanças são tão pequenas que é necessário amplificá-las para serem detectadas. Para isso, foi desenvolvido um índice que funciona comparando-se os parâmetros estimados e os reais. O AID calcula o índice comparando o valor estimado com o que está ocorrendo realmente. Se houver uma grande diferença, ele considera aquela área como provável local de ocorrência, porém, à fim de reduzir o número de alarmes falsos, é aplicado outro algoritmo como teste.

Já o algoritmo desenvolvido por Zhang et al. (2011) observa a ocorrência de um incidente devido à modificação dos parâmetros de tráfego nos carros de teste, como, por exemplo, uma redução brusca de velocidade. Com a finalidade de reduzir os falsos alarmes, o alarme final só é gerado após a observação das modificações dos parâmetros em mais de um carro de teste e por um tempo prolongado. Uma das vantagens desse método é o fato de não utilizar detectores fixos, o que diminui o alto custo dos detectores e as pequenas falhas na cobertura. Já uma das desvantagens é a demora na detecção, porém, mesmo assim, a metodologia se mostrou eficaz e aplicável na prática.

Zhao et al. (2010) também utilizaram veículos teste para obter os parâmetros de tráfego. A velocidade neste caso é calculada a partir do processamento dos dados obtidos. Depois é feita uma análise temporal, observando o momento em que a velocidade ficou abaixo do limite esperado. A seguir, é feita uma analise espacial, observando a diferença entre pontos adjacentes do arco de estudo. $\mathrm{O}$ alarme é acionado ao identificar a ocorrência de um incidente, se tanto os veículos-teste quanto a análise temporal de velocidade mostrar mudanças do padrão. Entretanto, o algoritmo fica prejudicado quando o número de veículos-teste naquela região é pequeno ou há poucos dados sobre incidentes.

Uma pesquisa mais recente apresentada por Kinoshita eu al (2015) utilizou carros de teste na coleta de dados com a finalidade de identificar eventos não usuais tentando distinguir o congestionamento usual dos incidentes. A metodologia proposta compara a velocidade do carro de teste em tempo real com a velocidade usual da via, definindo assim se há uma situação anormal e a estimativa do estado do tráfego. Nessa estimativa cada segmento de via é caracterizado pelo desempenho esperada naquele horário, além disso, foram propostos diversos métodos para quantificar a divergência entre o tráfego usual e o atual, e assim definir a ocorrência ou não de um incidente. Comparado a outros sistemas existentes notou-se que o método proposto distingue trajetórias que envolvem acidentes melhor que outros métodos. O próximo passo dessa pesquisa será desenvolver o algoritmo com a metodologia descrita.

Jinglei et al. (2011) obtiveram os dados por meio de câmeras de vídeo. O método desenvolvido relaciona o fluxo com as faixas de tráfego existentes, obtendo uma matriz que contém o fluxo médio e sua variação. Após a obtenção desses dados que relacionam espaço e tempo pelo processamento de imagens, o algoritmo desenvolvido classifica a condição do tráfego existente na via como: livre, bom, intenso ou congestionado.

Os algoritmos de detecção automática de incidentes desenvolvidos para túneis estudados utilizaram o processamento de imagens preocupando-se com a dificuldade de obter boas imagens, já que esse tipo de via tem baixa visibilidade.

Por meio das imagens detectadas no túnel de estudo, Kamijo e Fujimura (2010) buscaram uma detecção de incidente mais rápida em túneis urbanos com tráfego já saturado. Foi utilizado um algoritmo de rastreamento (tracking algorithm) com esse propósito. Resultando em um método que pode ser utilizado em vias congestionadas e com imagens precárias. O método em questão obtém a 
trajetória precisa dos veículos, observando assim a ocorrência de algum evento anormal na via. Uma das dificuldades encontradas é a calibração do método, porém, nos resultados de teste, o detector automático de incidentes desenvolvido se mostrou eficiente.

Já Schwabach et al. (2005) buscaram apenas definir os diversos cenários existentes nos túneis e colocar prioridades de detecção, além de buscar as tecnologias disponíveis de sensores de vídeo para ver se é possível aplicá-los no AID desenvolvido. Como resultado final, eles desenvolveram uma arquitetura de estudo e um protótipo do método denominado VITUS-1.

Três questões foram consideradas relevantes para a detecção de incidentes: detecção de um objeto, situação do tráfego e eventos de tráfego perigosos. Porém, nessa pesquisa os sensores só foram capazes de detectar os objetivos parados dentro do túnel, o fogo e a fumaça foram deixados para pesquisas futuras. Os dados de teste para calibração desse sistema foram obtidos a partir de um túnel na Áustria e envolvem dados como velocidade, fatos como carros na contramão e dados derivados desses outros dois.

Mais recentemente, $\mathrm{Gu}$ et al (2016) propôs utilizar a rede social Twitter na detecção de incidentes, esse método faz com que a detecção de incidente não dependa de detectores. A metodologia apresentada buscou rastrear, processar e filtrar os tweets público através de palavras chaves que representam um incidente de tráfego e depois são geolocalizados. O método foi testado em duas regiões metropolitanas como complementos dos sistemas de detecção já existentes e o resultado foi positivo, além disso, observou-se nos finais de semana há mais informações sobre incidentes no Twitter do que nos dias de semana e durante o dia há mais pessoas postando informações do que a noite.

No mesmo ano, Steenbruggen et al (2016) propuseram um sistema de detecção automática de incidentes através de dados de operadores de telefone celular. A ideia da pesquisa é utilizar os dados de telefonia em conjunto com outros sistemas de detecção de incidentes. A pesquisa descobriu que o uso de telefones celulares é fortemente afetado pelo fluxo de tráfego da rodovia e pela ocorrência de incidentes de trânsito. A principal limitação do estudo é que o intervalo de uma hora dos dados os torna bastante imprecisos.

\section{RESULTADOS E DISCUSSÃO}

Além dos parâmetros de fluxo de tráfego, a metodologia desenvolvida para a detecção de incidentes em um túnel urbano congestionado utilizou o conceito de ondas de tráfego. Esse conceito foi levado em consideração para tentar diferenciar quando as modificações bruscas na velocidade, densidade e fluxo são devidas a um incidente ou consequência de outros fatores.

\subsection{Cálculo de previsão das ondas de choque}

Para o cálculo da previsão do tempo que uma onda de choque leva para atingir certo ponto na rede viária será considerado o esquema apresentado na Figura 4.

Como é possível observar no esquema proposto, dois pontos foram destacados, um dentro da galeria e um na via fora do túnel, B e A, respectivamente. 


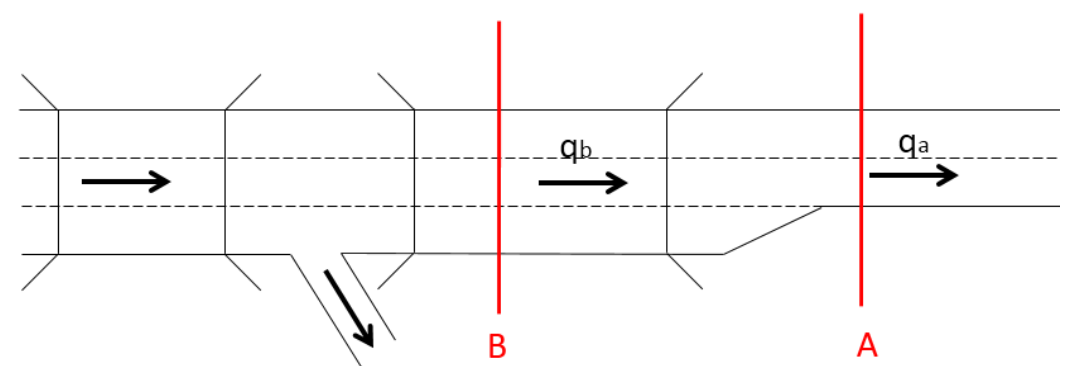

Figura 4- Esquema utilizado no método para cálculo da onda de choque Fonte: Elaborado pelos autores (2014)

As ondas de tráfego ocorrem quando a via atinge sua capacidade e passa a haver uma redução da velocidade e um aumento da densidade, formando as filas. A velocidade reduz até chegar a zero quando a densidade máxima é atingida (kj) (Figura 5). Caso o valor da densidade for superior a kcap, pode se dizer que a via atingiu a capacidade.

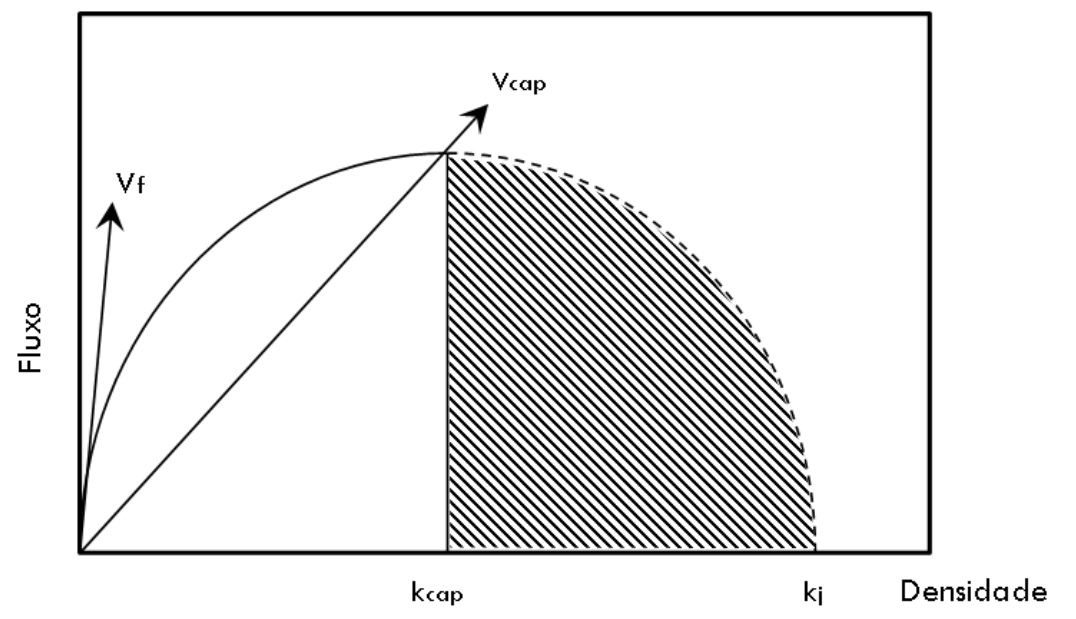

Figura 5- Relação entre o fluxo e a densidade Fonte: HCM (2010)

Sabendo que velocidade é a razão entre um deslocamento e o intervalo de tempo levado para efetuar esse deslocamento, pode-se prever o tempo que a onda de choque demora a chegar a certo ponto dentro do túnel por:

$$
t=\frac{\mathrm{d}_{\overrightarrow{\mathrm{AB}}}}{\mu}
$$

Em que: $\mu$ : Velocidade da onda de choque $(\mathrm{m} / \mathrm{s})$;

$$
\mathrm{d}_{\overrightarrow{A B}}: \text { Distância entre os pontos A e B (m). }
$$

Em situações normais, a velocidade nos pontos A e B seriam iguais, porém, a partir do momento que a velocidade em A fica menor que em $\mathrm{B}$, ocorrem ondas de choque que trazem instabilidade ao fluxo de tráfego em B.

Sendo $t_{0}$ o momento em que $V_{\alpha}<V_{b}$ e considerando $\mu=\mu_{k}$ calculado na Equação 1 , o instante $t_{A B}$ é definido por:

$$
t_{A B}=\frac{\mathrm{d}_{\overrightarrow{A B}}}{\left(\frac{\mathrm{q}_{\mathrm{b}}-q_{a}}{\mathrm{k}_{b}-\mathrm{k}_{a}}\right)}+t_{0}
$$


Pode haver ondas de choque consecutivas que atingem o ponto B. Sendo assim, é necessário prever todas as ondas de choque que podem atingir a galeria.

\subsection{Algoritmo desenvolvido}

Inicialmente devem-se definir dois pontos com distâncias entre si conhecidas. Nesses pontos devem ser levantados os parâmetros fluxo e densidade. São mostrados apenas dois pontos, A e B, conforme Figura 4, já que a lógica deve ser seguida em todos os trechos subsequentes.

Com os dados de fluxo e densidade é possível calcular a velocidade por meio da seguinte relação (HCM, 2010):

$$
\text { Velocidade }=\frac{\text { Fluxo }}{\text { Densidade }}
$$

Os dados de parâmetro de tráfego nos dois pontos devem ser obtidos no mesmo momento. Se a velocidade no ponto A for menor que a do ponto $\mathrm{B}$, a onda de choque irá se alastrar no sentido contrário ao fluxo, trazendo instabilidade no tráfego. pontos.

$\mathrm{O}$ valor de $t_{0}$ é o momento em que é observada a diferença de velocidade entre os dois

Devido à grande quantidade de dados obtidos em tempo real, é importante agrupar os parâmetros de tráfego de cada ponto em intervalos de tempo. Com os dados agrupados, é possível obter uma velocidade média e o fluxo em cada ponto naquele determinado intervalo.

Nesse intervalo de tempo $V_{a}<V_{b}$, deve-se prever o tempo gasto pela onda de choque para se alastrar até o próximo ponto, aplicando a equação 4 que relaciona distância com a velocidade. $\mathrm{O}$ tempo obtido deve ser somado ao tempo inicial da onda de choque, ${ }^{t_{0}}$, para obter o tempo previsto, $t_{A B}$, para chegar ao ponto B.

O tempo calculado para a onda de choque atingir o segundo ponto deve ser gravado, pois se naquele tempo guardado, $t_{A B}$, houver uma redução de velocidade no ponto $\mathrm{B}$, o programa não reconhecerá a mudança como um incidente.

Se $V_{a}=V_{b}$, a situação é considerada normal. Já se $V_{a}>V_{b}$, é preciso recuperar os tempos em que foi prevista a ocorrência de ondas de choque em $\mathrm{B},{ }_{A B}$, e comparar com o tempo atual. Se eles foram iguais, nada é acionado, já que era uma situação prevista. Se não houver nenhuma previsão para essa instabilidade, provavelmente há um incidente naquela região e um alarme é gerado.

O algoritmo desenvolvido é demonstrado de forma esquemática na Figura 6. 


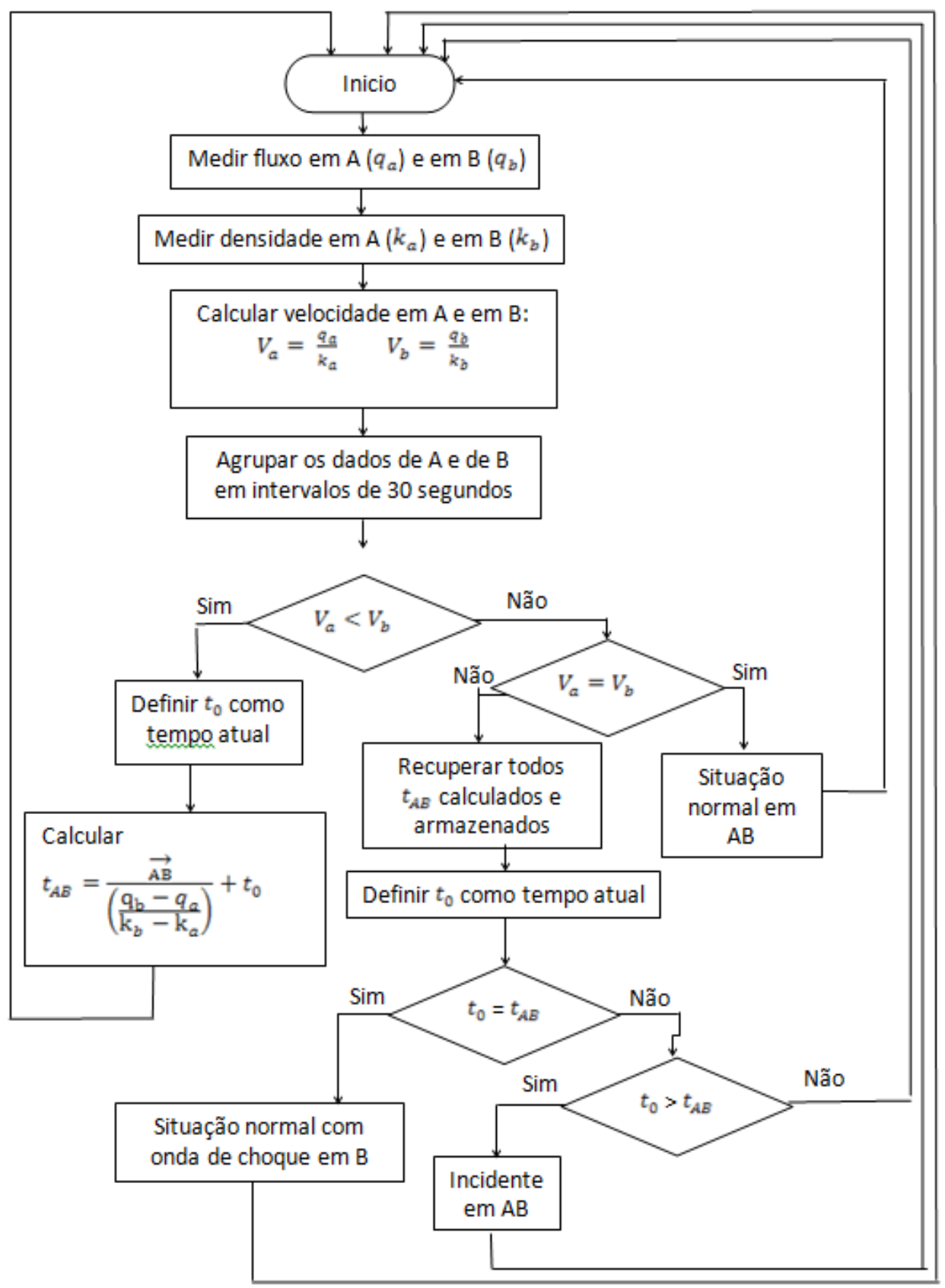

Figura 6- Algoritmo desenvolvido

Fonte: Elaborado pelos autores (2014)

\subsection{Estudo de caso}

O Túnel Rebouças foi escolhido para ser utilizado como estudo de caso do algoritmo proposto. Atualmente os incidentes são detectados por meio de um programa chamado Citilog (TRAFFICINFRATECH, 2012), que tem a finalidade de detectar situações anormais, sendo elas: parada com fluxo, parada com congestionamento, veículo lento e falta de visibilidade. Esse túnel foi o primeiro do país a ter sistema de detecção automática de incidentes.

Esse sistema ainda passa por calibração constante devido ao grande número de alarmes falsos que são gerados, principalmente nos horários de maior fluxo, quando ocorrem os congestionamentos. 
Segundo a SMTR - RJ, o Túnel Rebouças foi inaugurado em 03 de outubro de 1967, ligando as zonas Norte e Sul. Projetado para um volume de $76 \mathrm{mil}$ veículos/dia, com duas faixas de rolamento e acostamento, possui 2.040 metros de galeria contínua da Lagoa até o Cosme Velho e mais 772 metros na galeria do Cosme Velho até o Rio Comprido, totalizando 2.840 metros (Figura 7). Atualmente o volume diário é de $190 \mathrm{mil}$ veículos/dia, em três faixas de rolamento sem acostamento, com velocidade máxima permitida de $90 \mathrm{~km} / \mathrm{h}$.

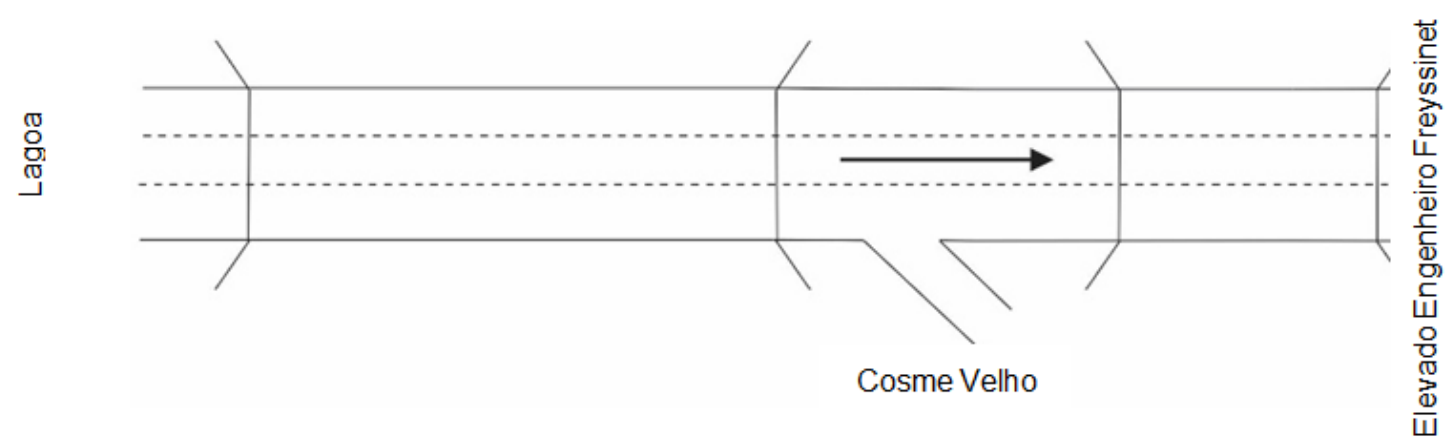

Figura 7- Esquema Túnel Rebouças

Fonte: Elaborado pelos autores (2014)

As galerias utilizadas para este estudo foram as do sentido Centro, que sofrem influência direta do Elevado Engenheiro Freyssinet, que possui apenas duas faixas de rolamento, fator que ocasiona modificação no fluxo, na velocidade e, consequentemente, na densidade nesse trecho.

Devido a dificuldades de se obter gravações dentro do túnel, foram utilizadas imagens obtidas pelas duas câmeras da CET-Rio localizadas no Elevado Engenheiro Freyssinet. Essas imagens foram gravadas em um dia de semana entre as 18:00 e 20:00 horas, horário de pico na via e com grande número de alarmes falsos no sistema de detecção automática de incidentes implantado dentro do túnel.

O headway e a densidade de cada ponto foram calculados a partir da sobreposição de figuras geométricas à imagem com a finalidade de ter sempre o mesmo ponto de referência para trabalhar com dados equivalentes. A densidade foi definida com a demarcação de uma área com $60 \mathrm{~m}$ de extensão nos vídeos, conforme Figura 8. A cada veículo que passava pela linha inferior do retângulo, eram contados quantos veículos havia naquela área e armazenados no Microsoft Office Excel.

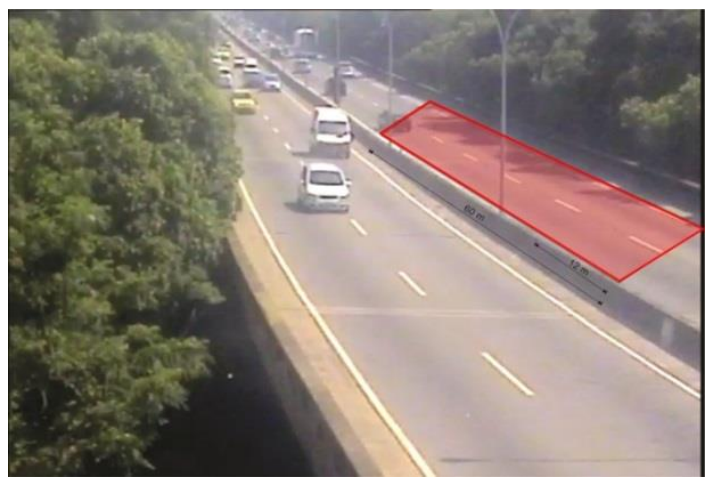

Figura 8- Área de cálculo da densidade FONTE: Câmera da CET-Rio

Para o cálculo do headway em segundos, foi calculada a diferença entre o tempo que cada veículo passava em uma linha predeterminada sobreposta às imagens das câmeras. Com esses dados, foi possível calcular o fluxo por meio da seguinte relação (HCM, 2010): 


$$
\text { Fluxo }=\frac{3600 s / h}{\text { Headway }}
$$

A velocidade foi obtida utilizando a Equação 4.

Após o cálculo dos parâmetros de tráfego de cada ponto separadamente, os dados obtidos com as imagens foram agrupados de $30 \mathrm{em} 30$ segundos. Com esses dados, foi montada uma planilha com o intervalo de tempo, a quantidade de veículos que passou em cada ponto naquele intervalo de tempo e a densidade.

Como a distância entre os dois pontos era conhecida (570 metros), foi possível calcular o tempo que demoraria uma onda de choque observada em A atingisse B. Esse tempo calculado somado ao tempo médio do intervalo de 30 segundos em questão é o momento previsto para haver instabilidade do tráfego no ponto B.

Para verificar se ocorreu instabilidade no tráfego no ponto B no tempo previsto, foi construído um gráfico que sobrepõe o tempo previsto à situação real. O gráfico mostrado na Figura 9 apresenta a velocidade das duas faixas de tráfego no ponto B (eixo vertical) em função do tempo (eixo horizontal) e junto a ele foram plotadas linhas tracejadas que ilustram o momento em que foram previstas ondas de choque.

Portanto, segundo os cálculos, é previsto que haja uma redução brusca na velocidade após as linhas tracejadas, independente da ocorrência de um incidente.

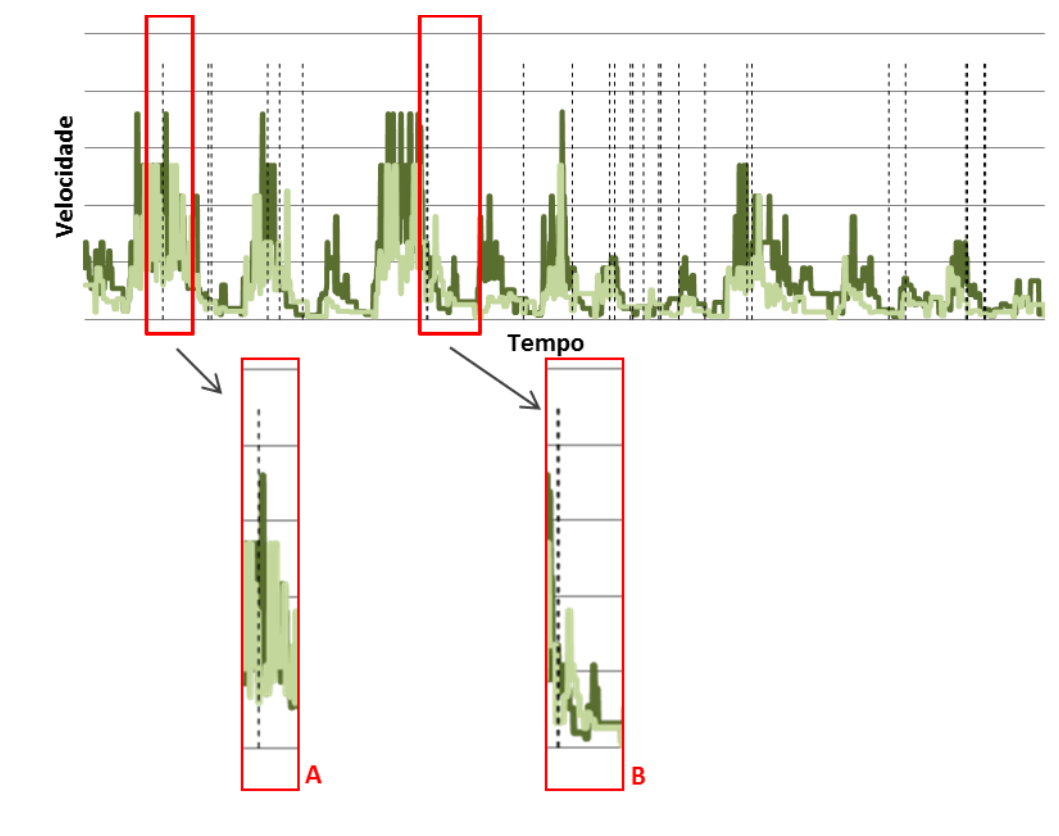

Velocidade na faixa 1

Velocidade na faixa 2

Figura 9- Velocidade no ponto B na pista sentido Centro Fonte: Elaborado pelos autores (2014)

Como essa análise foi feita no horário de pico da via, é notável a instabilidade da velocidade devido ao grande número de ondas de choque que atingem aquele ponto. Utilizando a metodologia desenvolvida, foi possível diferenciá-las de um incidente, já que as reduções bruscas já eram previstas (Figura 9). 
Anterior aos momentos em que a velocidade sofreu uma queda brusca há uma linha tracejada que mostra que essa mudança já era prevista e se trata de uma situação rotineira, como demonstrando no destaque B da Figura 9.

Em alguns pontos eram esperadas reduções de velocidades, mas elas não aconteceram, como mostrado no destaque A da Figura 9. Isso ocorre, provavelmente, devido à onda de recuperação que não foi levada em consideração nesse método.

Por extrapolação, foi calculado o tempo que as ondas de choque levariam para chegar a pontos predefinidos dentro do túnel. Como resumo desse cálculo, obteve-se a Tabela 1, que mostra a relação entre a velocidade da onda de choque e o tempo gasto para ocorrer modificações nos parâmetros de tráfego nas entradas e saídas das galerias do túnel.

Tabela 1:Relação entre a velocidade da onda de choque e o tempo que ela atingirá determinados pontos na via conforme esquema da Figura 7

\begin{tabular}{|c|c|c|c|c|}
\hline $\begin{array}{c}\text { Velocidade da } \\
\text { onda de choque } \\
\text { no ponto A (m/s) }\end{array}$ & $\begin{array}{c}\text { Tempo para } \\
\text { chegar ao } \\
\text { ponto B (s) }\end{array}$ & $\begin{array}{c}\text { Tempo para } \\
\text { chegar à saída } \\
\text { para Paulo de } \\
\text { Frontin (s) }\end{array}$ & $\begin{array}{c}\text { Tempo para } \\
\text { chegar à saída } \\
\text { do Cosme Velho } \\
\text { (s) }\end{array}$ & $\begin{array}{c}\text { Tempo para } \\
\text { chegar ao acesso } \\
\text { pela Lagoa (s) }\end{array}$ \\
\hline 2,5 & 228 & 548 & 636 & 1452 \\
\hline 5 & 114 & 274 & 318 & 726 \\
\hline 7,5 & 76 & 182 & 212 & 484 \\
\hline 10 & 57 & 137 & 159 & 363 \\
\hline 20 & 28,5 & 68,5 & 79,5 & 181,5 \\
\hline 30 & 19 & 45 & 53 & 121 \\
\hline
\end{tabular}

Fonte: Elaborado pelos autores (2014)

Esse método se diferencia dos outros pesquisados na revisão bibliográfica por considerar mais de uma variável de fluxo de tráfego e o fenômeno das ondas de choque a fim de diferenciar situações normais de tráfego das situações de incidentes. Sendo assim, o método torna mais confiável a detecção de incidentes em vias que trabalham em regime de saturação, isto é, com nível de serviço F.

\section{CONCLUSÃO}

O objetivo do trabalho foi alcançado após analisar os motivos que geram alarmes falsos nos sistemas de detecção automática de incidentes implantados em vias confinadas que já trabalham em regime de saturação, no caso, mais especificamente, em um túnel urbano.

Para reduzir os alarmes falsos nos sistemas existentes, foi proposto um método que busca prever as ondas de choque que podem atingir o túnel, diferenciando se a instabilidade do fluxo de tráfego está relacionada a um incidente na galeria ou se é apenas parada por congestionamento.

A metodologia foi desenvolvida de maneira genérica e pode ser utilizada em qualquer túnel ou via confinada que já trabalha em regime de saturação e é frequentemente atingida por ondas de choque. Sendo assim, é possível distinguir quando a via em questão foi atingida por uma onda de choque já prevista ou se ocorreu realmente um incidente.

Através do estudo de caso realizado no Túnel Rebouças observou-se que é possível obter uma redução dos alarmes falsos com a previsão das ondas de choque. $\mathrm{O}$ estudo de caso considerou duas câmeras em sequencia na via. Através dessas imagens e da metodologia desenvolvida foram calculados os tempos em que era esperada instabilidade de tráfego na montante. 
Para verificar se essa instabilidade no tráfego realmente ocorreu no ponto B no tempo previsto, foi construído um gráfico que sobrepõe o tempo previsto à situação real (Figura 9). Nesse gráfico comparativo notou-se que a metodologia já previa uma situação anormal no horário que ocorreu a instabilidade, sendo assim, aquela mudança abrupta nos parâmetros de tráfego não seria indicada como um incidente.

Como pesquisa futura, sugere-se a análise das ondas de recuperação, pois, em alguns momentos, a onda de recuperação pode ultrapassar a de congestionamento, não trazendo assim modificações aos parâmetros de tráfego na seção seguinte.

Como neste estudo de caso foram utilizados apenas dois pontos para análise, fica como recomendação testar a metodologia utilizando um maior número de câmeras, principalmente as internas, que mostram o comportamento do fluxo de tráfego dentro do túnel.

\section{REFERENCIAS}

CET-RIO. Relatório - Sistemas Inteligentes de Transportes CET-RIO. Prefeitura da Cidade do Rio de Janeiro, Rio de Janeiro, 2010

CHEN, L., CAO, Y., JI, R. Automatic Incident Detection Algorithm Based on Support Vector Machine. Revista IEEE, pp 864-866, 2010.

COELHO, E. C. Avaliação dos níveis de congestionamento em vias arteriais com a utilização da micro-simulação. Dissertação de M.Sc., Programa de Pós-graduação Engenharia de Transportes da Universidade Federal do Rio de Janeiro, Rio de Janeiro, RJ., Brasil, 2009.

GARBER, N.J E HOEL, L.A. Traffic and Highway Engeering. Second Edition. Revised Printing. PWS Publishing. 1996

GU, Y., QIAN, Z., CHEN, F. From Twitter to detector: Real-time traffic incident detection using social media data, Transportation Research Part C: Emerging Technologies, v.67, Junho de 2016, p. 321-342

HCM (2010) Highway Capacity Manual, Transportation Research Board, Washington D. C., USA.

IMMERS, L. H., LOGGHE, S. Traffic Flow Theory, Notas de aula sobre Teoria do fluxo de tráfego da Katholieke Universiteit Leuven, 2002.

JEONG, Y., CASTRO, M. E HAN, M. K. J. D. A wavelet-based freeway incident detection algorithm with adapting threshold parameters, Transportation Research Part C, n.19, p.1-19, 2011.

JIANG, G., NIU, S., LI, Q. CHANG, A. E JIANG, H. Automated Incident Detection Algorithms for Urban Expressway, Revista IEEE, v.3, p.70-74, 2010. Yantai, Shandong, Agosto.

JINGLEI, Z., JIN, X., E SHAOYI, L. Abnormal traffic incident detection based on hidden markov models", ICTE 2011, p.3098-3103.

KAMIJO E FUJIMURA. Incident Detection in Heavy Traffics in Tunnels by the Interlayer Feedback Algorithm”,Int. J. ITS Res, v.8, p. 121-130, 2010

KINOSHITA, A., TAKASU, A., ADACHI, J. Real-time traffic incident detection using a probabilistic topic model, Information Systems, v.54, p.169-188, Dezembro de 2015.

LOU, Y., YIN, Y. E LAWPHONGPANICH, S. Freeway service patrol deployment planning for incident management and congestion mitigation, Transportation Research Part C, v.19, p. 283-295, 2011

LU, J., CHEN, S., WANG, W. E RAN, B. Automatic traffic incident detection based on nFoil, Expert Systems with Applications, v.39, n.7, p.6547-6556, 2012.

SCHWABACH, H.,HARRER, M., HOLZMANN, W., BISCHOF, H., DOMÍNGUEZ, G., NÖLLE, M., PFLUGFELDER, R., STROBL, B., TACKE, A., WALTL, A. Video based image analysis for tunnel safety - vitus-1: a tunnel video surveillance and traffic control system, TRB, Fevereiro, San Francisco California, USA, 2005.

ŠKORPUT, P., MANDŽUKA, S. JELUŠIĆ:,N. Real-time Detection of Road Traffic Incidents”, Promet Traffic\&Transportation, v. 22, n. 4, p.273-283, 2010. 
SMTR-RJ. Disponível em <http://www.rio.rj.gov.br/web/guest/exibeconteudo?article-id=1838749>. Acesso em: 8 de setembro de 2011.

STEENBRUGGEN, J., Tranos, E. RIETVELD, P. Traffic incidents in motorways: An empirical proposal for incident detection using data from mobile phone operators, Journal of Transport Geography, v. 54, p. 81-90, Junho de 2016.

TRAFFICINFRATECH . Disponível em <http://www.citilog.com/pdf/en/TrafficTechMay2012.pdf〉. Acesso em maio de 2012.

TRB. Traffic Flow Theory, Washington D. C., Transportation Research Board, 1976.

VALENTI, G., LELLI, M. E CUCINA, D. A comparative study of models for the incident duration prediction. Department of Statistics, La Sapienza University, Roma, Italy, 2010.

WANG, Q. Traffic incident detection based on artificial neural network, Revista IEEE, 2011, p.657-659, 2011.

ZHANG, Z., LIN, X. E HU, B. Algorithm Design of Traffic Incident Automatic Detection Based on Mobile Detection, Revista IEEE, p.331 a 335, 2011.

ZHAO, X., WENG, J. E RONG, J. Urban Expressway Incident Detection Algorithm Based on Floating Car Data, Integrated Transportation Systems - Green Intelligent Reliable, p.2132 a 2139, 2010.

ZHENG, C., ZHOU, Q., CHEN,S. E YU,Z. Urban Road Traffic Incident Auto-detecting Based on Decision Fusion, ICCTP, p. 1348 a 1359, 2011. 